\title{
Methoden für die durchgängige Anwendung einer EBOM mithilfe klassenbasierter Substitutionsobjekte
}

Jonathan Leidich, Peter Robl, Julien Raphael Mrowka

Innerhalb der Initiative zur vierten industriellen Revolution, bei der Menschen, Produkt und Maschine in Echtzeit vernetzt sind, geht der Trend weg von der Serienfertigung und hin zur "Losgröße 1". Die ansteigende Anzahl an Varianten bringt jedoch auch höhere Verwaltungsaufwände mit sich. Um dem entgegenzuwirken wird die Methode der funktional substituierbaren Bauteile eingeführt. Diese ermöglicht die Verwaltung von technisch substituierbaren Komponenten innerhalb der Stücklisten einer konkreten Variante. Durch die Integration der Methoden FIM (Functional-Information-Model) und FIA (Functional-Information-Assembly) wird ein weiterer Schritt zur 3D-Modell-basierten Stückliste gemacht. Unter Zuhilfenahme dieser Methoden wird die notwendige Veränderung Darstellung der Struktur der im Produkt enthaltenen Bauteile während des Produktentstehungsprozesses beschrieben. Dabei werden die verwendeten Begriffe klar definiert und voneinander abgegrenzt.

Keywords: Digitalisierung, Stückliste, Variantenmanagement, 3D-Modell, EBOM, MBOM

\section{Aktuelle Anforderungen und Entwicklungspotentiale der Erstel- lung und Anwendungen von 3D-Modell-basierten Stücklisten}

Es gibt oft unzählige Varianten eines Produktes und bedingt durch die Globalisierung genauso viele Lieferanten in den unterschiedlichsten Nationen der Erde. Die Stückliste ist einer der substanziellsten Informationsträger im Produktlebenszyklus (PLC) und beinhaltet alle Bestandteile des Produktes inklusive der Stammdaten. (Eigner: 2014, S. 236ff.)

Auf der Basis der CAD-Struktur werden unterschiedliche Stücklisten-Sichten (OBOM, EBOM, MBOM) generiert. Wird die Stückliste darüber hinaus mit intelligenten Datenmodellen, wie bspw. dem FIM (Functional-Information-Model) oder FIA (Functional-Information-Assembly) assoziiert, so wird ein durchgängiges 3D-Modell-basiertes Arbeiten mit der Stückliste als führendes Ingenieursdokument gewährleistet. (Robl, Nowitschkow, Krause, Lipp, 2017) 
Neukonstruktionen und Kundenanpassungen bedingen im Umkehrschluss einen erhöhten Aufwand im Varianten- und Stücklistenmanagement.

In vielen Fällen ist keine Verknüpfung zwischen der Stückliste und den 3D-CAD-Modellen vorhanden. Bergriffe wie "Variante“ und "150\%-Stückliste" werden unterschiedlich interpretiert und angewendet. Bezeichnungen innerhalb des Varianten- und Stücklistenmanagement müssen allgemeingültig beschrieben werden. Funktional substituierbare Bauteile und Baugruppen innerhalb einer Stückliste müssen flexibel in die Stücklistenstruktur aufgenommen werden können. Die Verwaltung substituierbarer Objekte wird als integrierter Bestandteil der Stücklisten etabliert. Die wechselseitige Beziehung zwischen CAD-Struktur und Konstruktionsstückliste (als EBOM) muss eindeutig beschrieben sein. Im Folgenden werden die Begriffe, die im Kontext zur Beschreibung von Stücklisten angewendet werden, analysiert und festgelegt.

\section{$150 \%$ - und 100 \%-Konstruktionsstückliste (EBOM)}

Die Konstruktionsstückliste (en: Engineering Bill of Materials - EBOM) stellt die Sicht des Konstrukteurs auf ein Produkt dar und ist auftrags- und fertigungsneutral. Dadurch muss sie keine konkrete Erscheinung besitzen, sondern kann auch in Form einer Maximal- bzw. Variantenstückliste vorliegen. (DIN 6789) Sie ist funktional aufgebaut und spiegelt somit die Denkweise ihrer Anwender, dem Entwickler bzw. dem Konstrukteur, wider. Sie dient als Basis zur Erstellung der MBOM (en: Manufacturing Bill of Material). (Eigner, Stelzer: 2013, S.80)

Liegt die EBOM in Form einer Auswahl-Stückliste vor, so wird sie als „150 \%-EBOM“ bezeichnet. Existiert sie in konkreter Form (Produkt exakt definiert) einer Strukturstückliste, so wird von einer „100 \%-EBOM“ gesprochen. Die Auswahl-Stückliste, auch Komplex-Stückliste genannt, gehört zur Gruppe der Variantenstücklisten und eignet sich zur Darstellung sämtlicher Varianten eines Produktes. Folglich sind sämtliche Teile aller Produktvarianten in ihr enthalten, weshalb sie auch als $150 \%$-Stückliste bezeichnet wird. Die "100 \%-EBOM“ spiegelt eine funktional gegliederte, eindeutige Ausprägung (funktionale Variante) aus einer zugehörigen „150 \%-EBOM“ wider. (Wiendahl: 2014, S.163)

\section{Varianten des Produkts}

Obgleich der Begriff „Variante“ im generellen Sprachgebrauch häufig als selbsterklärend verstanden wird, weisen die Definitionen des Begriffs eine starke Heterogenität auf. Ganz allgemein wird eine „Variante" als „leicht veränderte Art oder Form von etwas" definiert (Kunkel-Razum 2020). Dieses „etwas“ wird aus technischer Sicht vom VDI 
als „eine Abart einer Grundausführung” konkretisiert, „wobei mindestens eine Merkmalsausprägung von der Grundausführung abweichen muss" (VDI 2815: 1978). Die Internationale Organisation für Normung (ISO) definiert den Begriff Varianten losgelöst von einer ursprünglichen Grundausführung als "Gegenstände ähnlicher Form und/oder Funktion mit, in der Regel hohem Anteil identischer Baugruppen oder Teile“ (DIN EN ISO 10209: 2012). Nach dieser Definition bedarf es keiner initialen Grundausführung, es müssen lediglich sich ähnelnde Ausführungen von Baugruppen oder Bauteilen vorliegen. Die Differenzierung zwischen den einzelnen Varianten eines Produktes muss hinsichtlich der Erfüllung der funktionalen Anforderungen der Baugruppe oder Bauteile geschehen. Unterschiedliche Lieferanten eines Bauteils erfüllen nicht automatisch den Variantenbegriff hinsichtlich der Funktion, da die Funktion des jeweiligen Bauteils unabhängig vom Lieferanten erfüllt sein muss. Es kann zusätzlich zwischen den Begriffen Produktvariante und Komponentenvariante unterschieden werden. Unter Komponentenvarianten werden Baugruppe oder Bauteile innerhalb eines übergeordneten Produkts verstanden, die in wenigstens einer Eigenschaft voneinander abweichen (Heiner Kesper: 2012, S.22).Da eine Komponentenvariante immer auch das zugehörige Produkt beeinflusst gibt es mindestens so viele Produktvarianten, wie Komponentenvarianten. Eine große Variantenvielfalt bewirkt einen hohen Individualisierungsgrad der Produkte und eine flexible Anpassung des Produktes an verschiedene Anforderungen bei gleicher Funktion.

\section{FSB - Funktional substituierbarer Baukasten}

Politische, soziale, ökologische, wirtschaftliche und qualitätsbezogene Aspekte führen dazu, dass unterschiedliche Lieferanten unterschiedliche Attraktivität beim Produktionsunternehmen bzw. dem jeweiligen Kunden besitzen, auch wenn die Geometrie und Funktion, unabhängig von den einzelnen Lieferanten der Produktkomponenten, gleichbleibt. Um diesen zunehmend bedeutenden Anforderungen in der Stückliste gerecht zu werden, wird die Methode des „Funktional substituierbareren Baukastens" (FSB) entwickelt. Das Prinzip lässt sich auf Komponenten anwenden, deren Substitution untereinander sich nicht auf die Charakteristika und Funktion des Produktes auswirken. Folglich werden durch eine Änderung der Komponentenwahl innerhalb eines FSB keine neue Funktionsvarianten geschaffen. Den verantwortlichen Konstrukteuren und dem strategischen Einkauf obliegt die Entscheidung über die Substituierbarkeit der Komponenten. Es können drei Arten von FSB unterschieden werden: einbaugleiche-, nicht einbaugleiche- und kombinierte FSB (Hermeler 2020). Repräsentanten innerhalb eines einbaugleiche FSB unterscheiden sich in ihren geometrischen Abmessungen höchstens marginal voneinander. Die Komponenten eines FSB können 
sich z.B. in Eigen- oder Fremdfertigung sowie im Fertigungsverfahren unterscheiden. Sind alle Komponenten des einbaugleichen FSB ausschließlich fremd gefertigt, wird hauptsächlich der jeweilige Hersteller differenziert. Die Komponenten aus diesem FSB lassen sich anwenden, ohne die Geometrie andere Bauteile verändern zu müssen oder auf die Funktion des Produktes Einfluss zu nehmen.

Nicht einbaugleiche FSB bestehen aus Komponenten, bei denen erst bei der Montage über die genaue Anzahl und Anordnung von Teilen entschieden werden kann. Nicht einbaugleiche FSB bestehen beispielsweise aus Distanzscheiben zum Ausgleich von Fertigungstoleranzen, die von einem Monteur nach Bedarf direkt bei der Montage ausgewählt werden. Da die angrenzenden Baugruppen und -teile nicht beeinflusst werden und die Funktion der Baugruppen gleichbleibt, sind die Teile beliebig substituierbar.

Kombinierte FSB sind eine Kombination der FSB-Arten einbaugleich und nicht einbaugleich. Öle und Fette mit gleichen Eigenschaften, die von verschiedenen Herstellern geliefert werden können, sind hierfür ein Musterbeispiel. Es kann zwischen verschiedenen Lieferanten gewählt werden und gleichzeitig kann erst bei der Montage die konkrete Menge des verwendeten Öls oder Fetts bestimmt werden.

Die Differenzierung eines FSB zu einer Variante wird durch zwei Aspekte festgelegt:

1. Konkrete funktionale Anforderung Werden die funktionalen Anforderungen von allen Komponenten gleichermaßen erfüllt, so liegt keine Variante vor. Ist die Anforderung an ein Fahrzeug bspw. "Metallic-Lackierung“ so könnten alle vorhanden Metallic-Lacke unter einem FSB geführt werden. Konkretisieren sich die Anforderungen jedoch bspw. durch die Anforderung "roter Metallic-Lack", so entstehen durch farblich unterschiedliche Metallic-Lacke (z.B. blau, schwarz) neue Varianten des Fahrzeuges.

2. Beeinflussung andere Komponenten im Produkt

Beeinflussen Komponenten angrenzende Baugruppe oder -teile verschiedenartig, so liegt selbst bei funktionaler Gleichheit, kein FSB vor. Müssen bspw. aufgrund der verschiedenen Einbaugrößen der Servomotoren, unterschiedliche Anpassungen an den angrenzenden Baugruppen oder -teilen gemacht werden (z.B. anderes Bohrbild oder Adapterplatte zum Ausgleich des Bohrbildes), so kann nicht mehr von funktional substituierbaren Komponenten gesprochen werden. Es entstehen also neue Varianten des Produktes. 


\section{Suffix zur Bezeichnung einer Stückliste „+“}

Um anzuzeigen, dass in einer Stückliste FSB enthalten sind, jedoch noch nicht konkretisiert vorliegen, wird das " ${ }^{\prime \prime}$ als Stücklisten-Suffix eingeführt. Eine "100 \%-EBOM+" ist demnach eine Konstruktionsstückliste einer konkreten Variante, in der mindestens ein FSB noch nicht konkretisiert ist. Sobald innerhalb jedes FSB eine konkrete Ausprägung selektiert wird, verschwindet das Suffix " ${ }^{+}{ }^{\prime}$. Durch das Konkretisieren der FSB wird keine neue Variante erzeugt. Liegen nichteinbaugleiche FSB vor, die meist erst in der Montage aufgelöst werden können, so wird aus der "100\%-EBOM+" eine "MBOM+" (en: Manufacturing Bill of Materials +) abgeleitet. Das heißt, das "+" bleibt so lange erhalten, bis die BOM eindeutig konkretisiert ist.

\section{FIM-, FIA-Methodik}

Die Methodik des Funktionalen-Informationsmodells (en: Functional Information Model FIM) baut auf der "Product and Manufacturing Information“ (PMI) auf und verfolgt das Ziel, funktionale Bauteilinformationen direkt im 3D-CAD-Modell zu hinterlegen und diese nicht erst manuell bei der Ableitung der 2D-Zeichnung zu erzeugen. Dadurch kann bereits in frühen Phasen des PEPs, im Vergleich zur herkömmlichen 2DZeichnungs-Erstellung, Zeit gespart und gleichzeitig die Grundlage für eine durchgängige 3D-CAD-Modell-Nutzungs-Kette geschaffen werden. Die automatisch abgeleiteten geometrischen Informationen können innerhalb der Prozesskette in einem Betrieb von verschiedenen Teilprozessen wie der Arbeitsplanung, CNC-, CMM- und CAM-Programmierung interpretiert und genutzt werden (siehe Abbildung 1). Haben bestimmte Montage- und Fertigungsparameter (z.B. ein Anzugsmoment) innerhalb des Herstellungsprozesses maßgeblichen Einfluss auf die späteren funktionalen Eigenschaften des Produktes, so ist es die Aufgabe der Entwicklung und Konstruktion diese explizit vorzugeben. Diese Informationen beziehen sich auf eine Gruppe von Bauteilen und können demnach nicht in einem bauteilspezifischen Informationsfeature im FIM gespeichert werden. An dieser Stelle kommt das Konzept der Funktionalen-Informationsbaugruppe (en: Functional Information Assembly FIA) zur Anwendung. Das FIA besteht aus einzelnen FIMs und verbindet mittels eines virtuellen Baugruppenknotens diejenigen Bauteile, unter denen relevante technische oder wirtschaftliche Wechselwirkungen existieren. (Robl, Nowitschkow, Krause, Lipp: 2017)

Mit dieser Methode können Fertigungsinformationen schnell und konsistent im 3DCAD-Objekt (der Baugruppe) bereitgestellt werden. Im Zuge dessen können FIM und FIA die 2D-Zeichnung, zur Produktdefinition, vollständig ablösen. Die Verknüpfung von FIM/FIA mit dem anwendungsspezifisch strukturierten Stücklistenaufbau (z.B. EBOM oder MBOM) schafft die Basis für eine 3D-basierte Prozesskette. Im Verbund mit FSB 
werden somit 3D-Modell-basierte, flexible und anwendungsbezogene Stücklistenstrukturen geschaffen.

\section{Dokumentenstammsatz und Artikelstammsatz als Beschreibungsbasis}

Um 3D-Modell-basiertes Arbeiten mit der Stückliste zu realisieren, müssen die jeweiligen Artikel- und Dokumentenstrukturen verknüpft werden. Dies erfolgt durch eine Verknüpfung der jeweiligen Artikel- und Dokumentenstammsätze.

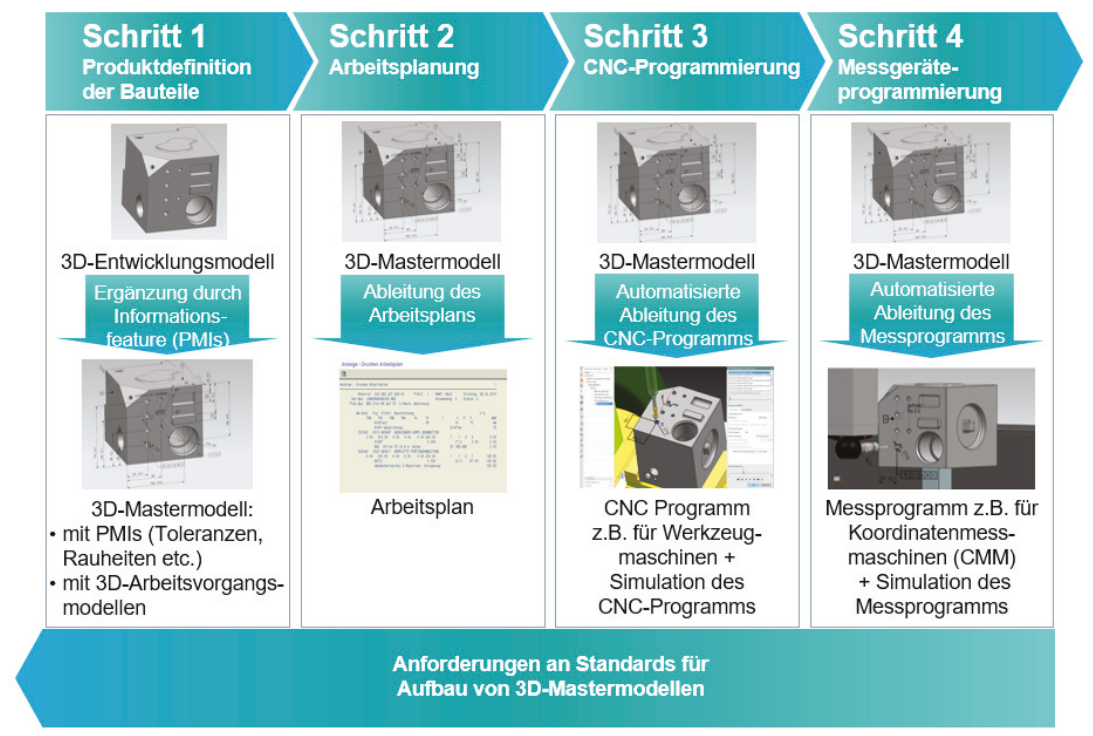

Abbildung 1: Durchgängige 3D-CAD-Modell-Nutzungs-Kette (Neubert und Robl: 2018)

\section{Artikelstammsatz (ASS)}

Der Artikelstammsatz (ASS), im SAP-Fachjargon auch oft als Materialstammsatz bezeichnet, ist ein Datensatz, der alle Meta-Information beinhaltet, die in sämtlichen Unternehmensabteilungen gelten und zur Verwaltung des jeweiligen Artikels vonnöten sind (Eigner,Stelzer: 2013, S.95). Der ASS beinhaltet eindeutig identifizierende und klassifizierende Eigenschaften (Merkmale) des Artikels wie z.B. Artikel-ID, Benennung, Werkstoff, Hersteller oder Masse. Um eine konsistente Datenverwaltung zu erreichen, muss jeder Artikel einen ASS besitzen. Ein Artikel kann mit verschiedenen gültigen Dokumenten (z.B. einem 3D-CAD-Modell, einer 2D-Zeichnung, einem Versuchsbericht) beschrieben sein. 


\section{Dokumentenstammsatz (DSS)}

Analog zu einem Artikel besitzt auch ein Dokument einen Stammsatz, der als Dokumentenstammsatz (DSS) bezeichnet wird. Um eine effektive Dokumentenverwaltung zu erreichen, werden Dokumente, die in einem Verwaltungssystem gespeichert sind, analog dem Artikelstammsatz, mit Dokumentenstammdaten bzw. Metadaten, in Form von identifizierenden und beschreibenden Merkmalen, konkretisiert. Durch das Festlegen von identifizierenden Merkmalen, wie bspw. der Dokumentennummer und dem Revisionsstand des Dokumentes, wird dieses eindeutig identifiziert. Ein Dokument kann Verknüpfungen zu einem oder mehreren Produkten/Bauteilen bzw. deren Artikeln haben. (Volker: 2011, S.106)

\section{Durchgängige Nutzung von 3D-ProduktDaten innerhalb einer flexiblen, integrierten Stückliste}

Durch die Gliederung der EBOM nach dem funktionalen Denken des Entwicklers oder Konstrukteurs, wird ein flexibles Arbeiten mit der Stückliste im späteren Produktionsprozess erforderlich. Mit Hilfe der FIM- und FIA-Integration wird ein weiterer Schritt hin zu einer durchgängigen Prozesskette, die auf den 3D-CAD-Modellen basiert, gemacht.

\section{Erstellung der EBOM auf Basis der CAD Struktur}

In Abbildung 2 wird der Prozess der Ableitung der Montagestruktur aus einer funktional aufgebauten CAD-Struktur dargestellt. Dabei sind das Variantenmanagement und die Möglichkeit der Nutzung von FSB integraler Bestandteil.

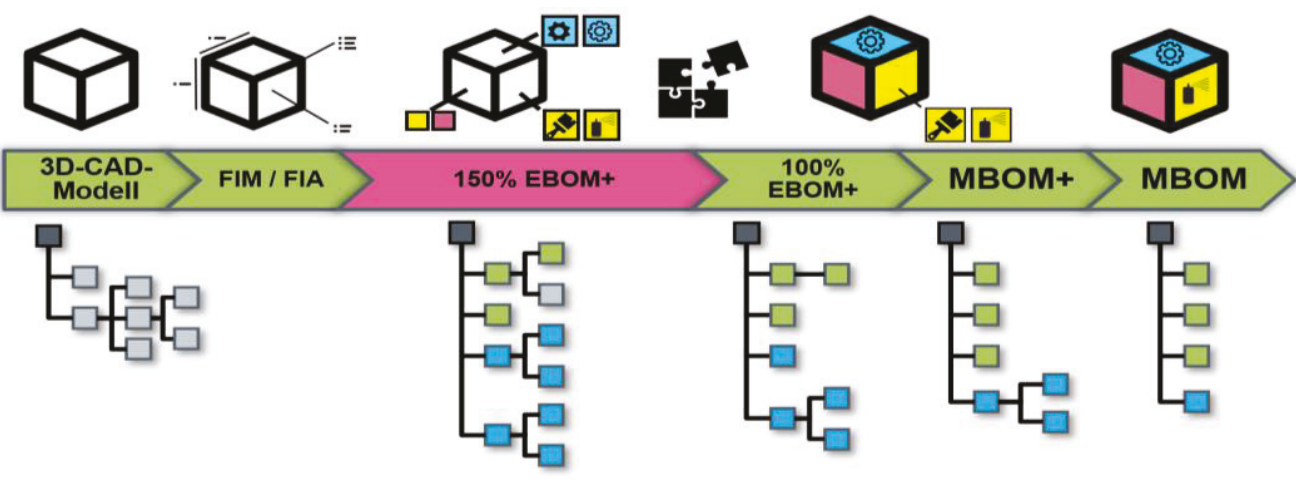

Abbildung 2: Durchgängige Produkt- und Prozessdatennutzung und

Produktstrukturänderungen während des PEP 
Die 3D-CAD-Geometrie-Modelle in einer Baugruppe besitzen bereits Beziehungen untereinander die in Form der CAD-Struktur vorliegen. Da die 150 \%-EBOM+ alle Varianten eines Produktes abdecken soll ist es notwendig, mehrere CAD-Strukturen zu bündeln. Jedoch ist das zwanghafte Bündeln von stark abweichenden Varianten innerhalb einer Maximalstückliste nicht immer sinnvoll. Daher ist die Bildung von VariantenStrukturen auf die funktionale Gleichheit der Objekte zu beziehen. Tabelle 1 stellt Vorund Nachteile einer 150 \%-EBOM dar, die bei einer möglichen Einführung gegeneinander abgewogen werden müssen.

Tabelle 1: Vor- und Nachteile einer $150 \%$-EBOM

Vorteile einer $150 \%$-EBOM

Nur eine einzige Stückliste als Basis für den Konfigurationsprozess notwendig

Alternativkomponenten können besser miteinander verglichen werden
Nachteile einer $150 \%$-EBOM

Schwer darstellbar, wenn die Baugruppenstruktur sich von Variante zu Variante unterscheidet

Erhöhter Verwaltungsaufwand durch das Erstellen und Pflegen einer 150 \%-EBOM

Unabhängig davon, ob eine 150 \%-EBOM gebildet wird, müssen Artikel, die kein 3DCAD-Modell besitzen, was bspw. bei Lacken, Ölen oder Klebstoffen der Fall ist, mit einem oder mehreren anderen deskriptiven Dokumenten vernetzt und der Stückliste hinzugefügt werden. Um eine Basis für die Erstellung einer klar abgegrenzten und funktionsmodularisierten Stückliste zu schaffen, müssen zusätzlich alle für das Produkt benötigten Funktionsklassen innerhalb eines Klassifizierungssystems festgelegt sein. Da eine Klassifizierung via universeller Klassifizierungssysteme (z.B. ecl@ss (Volker: 2011, S.145)) meist ungeeignet zur Beschreibung von produktspezifischen Funktionsbaugruppenklassen ist, sollten diese vom Unternehmen selbst möglichst konkret festgelegt und aufgebaut werden. Alle Objekte der CAD-Struktur (Einzelteile und Baugruppen) sowie die Objekte der Stücklistenstruktur eineindeutige Dokumenten- bzw. Artikelnummern. Die CAD-Struktur stellt eine Struktur aus 3D-CAD-Dokumenten dar, während die Stückliste die Struktur der Artikel des Produktes vorgibt. Das Erzeugen von "klickbaren" Verlinkungen zwischen Artikel und Dokument, stellt eine Standardfunktion gebräuchlicher PDM-Systeme dar, wodurch das 3D-CAD-Modell-basierte Arbeiten mit der Stückliste gewährleistet wird. 


\section{Integration von FIM und FIA}

Maße und Toleranzen sowie viele weitere Informationen, wie z.B. eine Gefahrstoffkennzeichnung, eine Härte- oder Beschichtungsanforderung oder eine Oberflächenangabe, werden in Form von Informationsfeatures im FIM bzw. FIA gespeichert (Robl, Nowitschkow, Krause, Lipp: 2017). Wichtig dabei ist, dass das initiale 3D-CAD-Modell und das darauf aufbauende FIM, dasselbe Datenobjekt ist, welches sich während des PLC ändert bzw. weiterentwickelt. Die Dokumentennummer des 3D-CAD-Modells, des FIM, bleibt somit immer dieselbe und unterliegt dem Änderungswesen. In Abbildung 3 ist ein FIA dargestellt. Es kann nun eine Information zur Beziehung (Freimaßtoleranz) des Zahnrads (orange) und der Welle (grau) in Form eines PMI hinterlegt werden.

Außerdem ist zu erkennen, dass ein FSB innerhalb der CAD-Struktur nur durch einen Repräsentanten des FSB (lila Zahnrad) vertreten wird und erst in der EBOM aufgegliedert integriert wird.

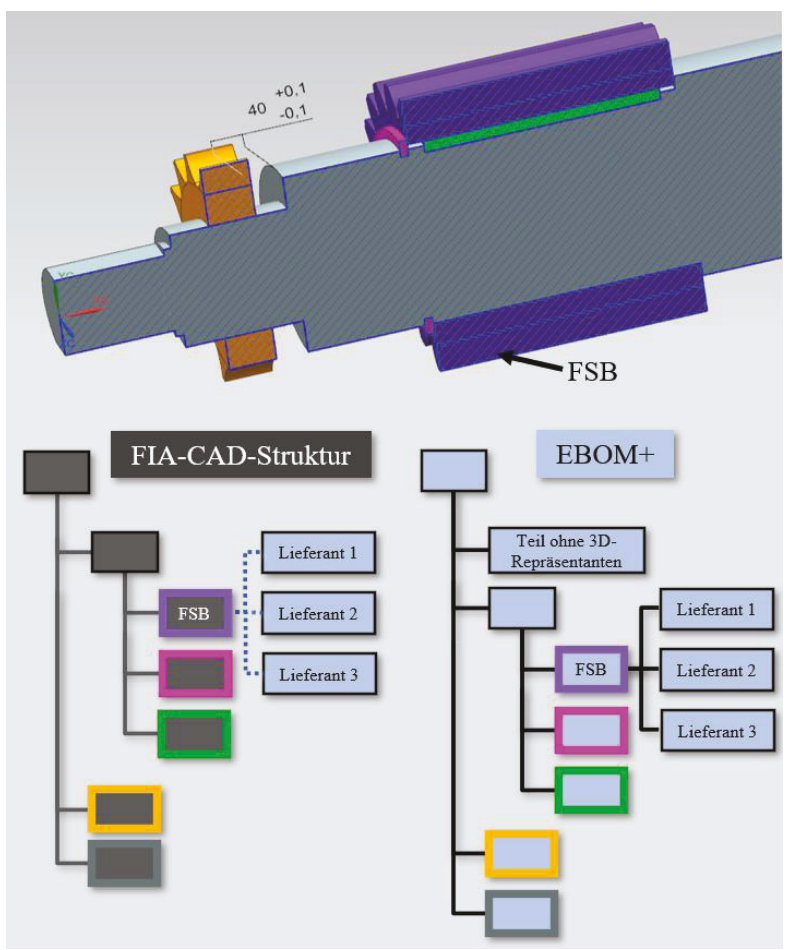

Abbildung 3: Konzept der agilen, zukunftsfähigen Stückliste auf Basis der FIA-CAD-Struktur 


\section{EBOM mit FSB und Ableitung der MBOM}

Werden alle erforderlichen bzw. möglichen FSB von der Konstruktionsabteilung angelegt, kann eine funktional modularisierte $150 \%$-EBOM+ erstellt werden. Die Stückliste $(150 \%-E B O M+)$ in Abbildung 2 besitzt bspw. einen einbaugleichen FSB (z. B. ein Kugellager von mehreren verschiedenen Herstellern) und einen kombinierten FSB (Öl von verschiedenen Herstellern, dessen Menge erst bei der Befüllung bestimmt werden kann). Im Konfigurationsprozess wird zwar funktional das Kugellager festgelegt, der Hersteller aber offengelassen. Es entsteht also eine "100 \%-EBOM+“ mit dem Kugellager als FSB. Bei der Übertragung dieser "100\%-EBOM+“ in das ERP-System kann nun Z. B. vom Einkauf der Hersteller des Kugellagers festgelegt werden und eine $100 \%$ MBOM abgeleitet werden. Anders verhält es sich beim Beispiel mit dem Öl. Der Einkauf kann den Hersteller des Öls vor der Montage bestimmen und am Befüllort bereitstellen. Die Menge des Öls wird aber vom Monteur beim Befüllen festgelegt (z. B. „Befüllung bis zu einer bestimmten Markierung"). Im Entwicklungsprozess wird die $100 \%$ EBOM oder $100 \%$-EBOM+ der Produktionsplanung übergeben, die daraus eine MBOM ableitet, die wiederum als Ausgangspunkt für die Terminierung und der anschließenden Montage ist. Da die MBOM hochgradig von lokalen Gegebenheiten des Shopfloors abhängt und sich daher auch enorm von der EBOM (z. B. in der Struktur) unterscheiden kann, muss die Ableitung der MBOM standortindividuell erfolgen. Bei der Ableitung der MBOM wird die EBOM so umstrukturiert, wie es die lokalen Vormontage- und Hauptmontageprozesse erfordern. Die EBOM fungiert also als Quelle aller zu montierenden Einzelteilen. (Stekolschik 2017)

\section{Zusammenfassung/Fazit}

Die Klassifikation von Bauteilen und Baugruppen nach ihren geometrischen Ausprägungen bzw. funktionalen Eigenschaften ist eine Grundlage für ein effektives Variantenmanagement. Die Methode zum Einsatz von funktional substituierbaren Komponenten oder Baugruppen ist ein Baustein, um die Variantenflut und die Handhabung in der Fabrik in den Griff zu bekommen. Das 3D-CAD-Modell als FIM oder FIA wird die 2D-Zeichnung ablösen und so einen weiteren Bruch der Prozesskette schließen. In Kombination bilden die Methoden die Basis für ein durchgängiges 3D-Modell-basiertes Arbeiten mit der Stückliste in den unterschiedlichen Bereichen eines Unternehmens. 


\section{Literaturverzeichnis}

DIN 6789. Dokumentationssystematik - Verfälschungssicherheit und Qualitätskriterien für die Freigabe digitaler Produktdaten.

DIN EN ISO 10209 (2012). Technische Produktdokumentation - Vokabular - Begriffe für technische Zeichnungen, Produktdefinition und verwandte Dokumentation.

Eigner, Martin (2014). Modellbasierte virtuelle Produktentwicklung.

Eigner, Martin/Stelzer, Ralph (2013). Product Lifecycle Management. Ein Leitfaden für Product Development und Life Cycle Management. 2. Aufl. Dordrecht, Springer.

Heiner Kesper (2012). Gestaltung von Produktvariantenspektren mittels matrixbasierter Methoden.

Kunkel-Razum, Kathrin (2020). Duden - Die deutsche Rechtschreibung. Auf der Grundlage der aktuellen amtlichen Rechtschreibregeln. 28. Aufl.

Lukas Hermeler (2020). Entwicklung eines Methodenbaukastens zur Erstellung einer 3D-Modell-basierten Konstruktionsstückliste mit der Möglichkeit funktions- und einbaugleiche Teile zu substituieren. Masterarbeit. Bochum, Ruhr Universität Bochum.

Neubert, Sebastian Dr./Robl, Peter Dr. 3D-CAD-Modelle lenken die Fertigung. In: VDI-Z Integrierte Produktion.

Prof. Alexander Stekolschik (2017). Methods for automated semantic definition of manufacturing structures (mBOM) in mechanical engineering companies, 2017.

Robl, Peter/Nowitschkow, Alexander/Krause, Stefan/Lipp, Christian. Functional Information Model - FIM. Patentschrift, Veröffentlichungs-Nr.: 18171518.6-1224, Deutschland.

Volker, Arnold (2011). Product Lifecycle Management beherrschen. Ein Anwenderhandbuch für den Mittelstand. 2. Aufl. Berlin, Springer.

Wiendahl, Hans-Peter (2014). Betriebsorganisation für Ingenieure. 8. Aufl. München, Hanser.

\section{Kontakt}

Dr. Peter Robl (peter.robl@siemens.com)

Jonathan Leidich (jonathan.leidich.ext@siemens-energy.de)

Julien Mrowka (Julien.Mrowka@siemens.com)

Siemens AG

81739 München 